\title{
Preface
}

\section{The Costly Face of Sleep Disorders}

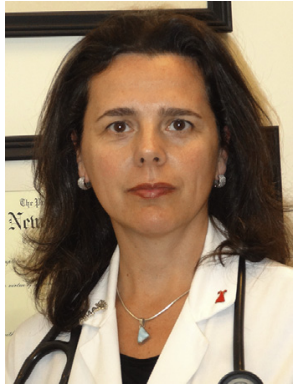

Ana C. Krieger, MD, MPH, FAASM, FCCP Editor

Welcome to the March 2017 issue of the Sleep Medicine Clinics. This issue is dedicated to reviewing the social and economic impacts of sleep disorders. The importance of sleep has become a topic of frequent discussion in the literature. The progress experienced over the past century, including an increased availability of artificial light, easy access to processed and manufactured foods, improved communication, and transcontinental travel, has fundamentally altered our daily activities and clearly impacted sleep physiology. Modern societies are now facing challenges in achieving adequate sleep and experiencing a multitude of sleep disturbances.

Technological advances have been, nonetheless, key to scientific knowledge expansion and research development. Since the discovery of electroencephalography almost a century ago, the field of Sleep Medicine has grown exponentially, moving from obscurity to a well-known multidisciplinary medical specialty. Sleep disorders have become more easily identified, and new treatment approaches have been developed. With an estimated prevalence of more than $30 \%$ of the adult US population, sleep disturbances are associated with an increased burden to the health care and financial systems, carrying intrinsic costs to the individual and society in general.

In this issue of Sleep Medicine Clinics, an outstanding group of collaborators was gathered to discuss the socioeconomic implications of disrupted sleep. The topics presented include a review of the current state of sleep health, the impact of pediatric and geriatric sleep disturbances, as well as the costs associated with insomnia, hypersomnia syndromes, and narcolepsy in our society. The reader will also find articles on the impact of specific neurologic, cardiac, and respiratory disorders in sleep, and on the application of perioperative screening and portable testing, closing with an overview of the legal and regulatory framework in sleep medicine.

Thank you for your interest in this publication. We hope the information shared will help to better understand the implications of sleep disturbances, and the pressing need to improve sleep for all.

I would like to express my gratitude to the dedicated colleagues that contributed to this issue, and I wish you all a healthy journey toward optimal sleep.

Ana C. Krieger, MD, MPH, FAASM, FCCP

Weill Cornell Medicine/

New York-Presbyterian Hospital Departments of Medicine, Neurology and Genetic Medicine Weill Cornell Medical College

Cornell University

425 East 61st Street-5th Floor New York, NY 10065, USA

E-mail address: ack2003@med.cornell.edu 